\title{
Liver Congestion in Heart Failure Contributes to Inappropriately Increased Serum Hepcidin despite Anemia
}

\author{
Yukako Ohno, ${ }_{1}^{1}$ Haruo Hanawa, ${ }^{1}$ Shuang Jiao, ${ }^{1}$ Yuka Hayashi, ${ }^{1}$ Kaori Yoshida, ${ }^{1}$ \\ Tomoyasu Suzuki, ${ }^{1}$ Takeshi Kashimura, ${ }^{1}$ Hiroaki Obata, ${ }^{1}$ Komei Tanaka, ${ }^{1}$ \\ Tohru Watanabe ${ }^{1}$ and Tohru Minamino ${ }^{1}$ \\ ${ }^{1}$ Department of Cardiovascular Biology and Medicine, Niigata University Graduate School of Medical and Dental \\ Sciences, Niigata, Niigata, Japan
}

\begin{abstract}
Hepcidin is a key regulator of mammalian iron metabolism and mainly produced by the liver. Hepcidin excess causes iron deficiency and anemia by inhibiting iron absorption from the intestine and iron release from macrophage stores. Anemia is frequently complicated with heart failure. In heart failure patients, the most frequent histologic appearance of liver is congestion. However, it remains unclear whether liver congestion associated with heart failure influences hepcidin production, thereby contributing to anemia and functional iron deficiency. In this study, we investigated this relationship in clinical and basic studies. In clinical studies of consecutive heart failure patients $(n=320)$, anemia was a common comorbidity $(41 \%)$. In heart failure patients without active infection and ongoing cancer $(n=30)$, log-serum hepcidin concentration of patients with liver congestion was higher than those without liver congestion $(p=0.0316)$. Moreover, in heart failure patients with liver congestion $(n=19)$, the anemia was associated with the higher serum hepcidin concentrations, which is a type of anemia characterized by induction of hepcidin. Subsequently, we produced a rat model of heart failure with liver congestion by injecting monocrotaline that causes pulmonary hypertension. The monocrotaline-treated rats displayed liver congestion with increase of hepcidin expression at 4 weeks after monocrotaline injection, followed by anemia and functional iron deficiency observed at 5 weeks. We conclude that liver congestion induces hepcidin production, which may result in anemia and functional iron deficiency in some patients with heart failure.
\end{abstract}

Keywords: anemia; heart failure; hepcidin; iron deficiency; liver congestion

Tohoku J. Exp. Med., 2015 January, 235 (1), 69-79. (C) 2015 Tohoku University Medical Press

\section{Introduction}

Anemia frequently occurs in patients with heart failure (HF) and is an independent prognostic factor for mortality (Ezekowitz et al. 2003; Hamaguchi et al. 2009). The etiology of anemia in HF patients is thought to be multifactorial. Hemodilution (Androne et al. 2003), chronic inflammation (Weiss and Goodnough 2005), renal dysfunction (Eschbach 2002), hemolysis (Kliger et al. 2013), gastrointestinal bleeding (Holleran et al. 2013), bone marrow dysfunction (Westenbrink et al. 2010), resistance to erythropoietin (van der Meer et al. 2008), hematinic deficiencies including vitamin B12, folic acid, and functional iron deficiency (FID) (Witte et al. 2004; Opasich et al. 2005) are thought to be etiological factors of anemia in HF patients. Especially, absolute and FID have been reported to be an important factor and a new term, cardiorenal-iron deficiency syndrome was proposed (Macdougall et al. 2012).

The central player regulating the amount of iron in the body is hepcidin (Ganz 2003). Therefore, hepcidin is postulated to play an important role in anemia and FID in HF (Matsumoto et al. 2010; Jankowska et al. 2013). Hepcidin is mainly produced in hepatocytes and hepcidin excess causes iron deficiency and anemia by inhibiting iron absorption from the intestine and iron release from macrophage stores (Ganz 2003). Therefore, we focused on pathological findings of liver in HF with anemia. The most frequent histologic appearance of liver in HF patients is congestion. We hypothesized that liver congestion (LC) in HF patients may influence expression of hepcidin and increased expression of hepcidin may contribute to anemia and FID.

Hepcidin expression is induced and regulated by multiple factors (Fleming and Ponka 2012). Anemia and hypoxia reduce production of hepcidin (Nicolas et al. 2002), but on the other hand, increase of serum holo-transferrin and iron concentration in cells induce hepcidin expression via bone morphogenetic protein-6 (Arndt et al.

Received October 20, 2014; revised and accepted January 6, 2015. Published online January 24, 2015; doi: 10.1620/tjem.235.69.

Correspondence: Haruo Hanawa, M.D., Department of Cardiovascular Biology and Medicine, Niigata University Graduate School of

Medical and Dental Sciences, 1-757 Asahimachi-dori, Chuo-ku, Niigata, Niigata 951-8510, Japan.

e-mail: hanawa@med.niigata-u.ac.jp 
2010). Inflammation also induces hepcidin expression via interleukin-6 (Nemeth et al. 2003). Previously, we reported that a patient with severe tricuspid regurgitation with $\mathrm{LC}$, refractory anemia and a very high serum hepcidin level recovered from anemia after valve replacement, which was accompanied by a decrease in the hepcidin and interleukin-6 levels (Suzuki et al. 2012). Moreover, we demonstrated that LC by ligation of the inferior vena cava (IVC) of rats caused inappropriately increased expression of hepcidin via bone morphogenetic protein- 6 and interleukin- 6 and contributed to anemia and FID (Suzuki et al. 2014). These results suggested that LC may contribute to anemia and FID of HF via inappropriately increased expression of hepcidin.

Contribution of LC to anemia and FID in HF has not been investigated. It remains unclear whether inappropriately increased expression of hepcidin occurs in HF patients with LC. In this study, we tested the hypothesis in HF patients admitted to the Cardiovascular Disease Department of Niigata University Medical and Dental Hospital. In addition, pulmonary arterial hypertension (PAH) generally leads to right HF and sometimes cause to LC (Alvarez and Mukherjee 2011). It has been reported that inappropriately raised hepcidin levels may be a causative factor for FID of patients with PAH (Rhodes et al. 2011), but the mechanisms of increased hepcidin levels in PAH are largely unknown. Therefore, to address these questions, we also studied these interrelationships using a rat model of PAH.

\section{Methods}

\section{Patient samples}

In clinical study 1, we aimed to examine the prevalence of anemia in all HF patients admitted to the Cardiovascular Disease Department of Niigata University Medical and Dental Hospital for one year, and the relationship between markers of HF and markers of anemia in all HF patients was evaluated. Blood samples were obtained from consecutive patients $(n=320)$ with HF admitted between August 2013 and July 2014. HF was defined as a brain natriuretic peptide (BNP) concentration of $50 \mathrm{pg} / \mathrm{mL}$ or higher and we defined HF only based on the BNP value. Blood hemoglobin $(\mathrm{Hb})$ concentration of all patients was determined. Anemia was defined as a $\mathrm{Hb}$ concentration below the lower level of normal (male, $\mathrm{Hb}<13.2$ $\mathrm{g} / \mathrm{dL}$; female, $\mathrm{Hb}<10.8 \mathrm{~g} / \mathrm{dL}$ ), and then serum iron and unsaturated iron-binding capacity were determined in as many patients with anemia as possible. The IVC diameter, an indicator of LC, was measured at the end expiratory phase by ultrasound examination.

In clinical study 2, we aimed to examine the influence of LC on serum hepcidin levels. Blood samples were obtained from patients with anemia and HF $(\mathrm{BNP}>50 \mathrm{pg} / \mathrm{mL})(n=45)$, who gave their consent and were admitted to the Cardiovascular Disease Department of Niigata University Medical and Dental Hospital between 2008 and 2013. The IVC diameter was measured at end expiratory phase by ultrasound examination. LC was diagnosed with IVC diameter $(\geq 2.0$ $\mathrm{cm})$ from echography findings of patients. Tricuspid regurgitation pressure gradient (TRPG) can be measured for 38 patients. Because anemia of chronic diseases, such as active inflammation and noncurative cancer, is also caused by hepcidin (Weiss and Goodnough
2005), we excluded patients with chronic disease such as autoimmune disease, active infection, and ongoing cancer $(n=15)$, and analyzed the remaining patients $(n=30)$ for further analyses of serum hepcidin levels. Autoimmune disease, active infection and ongoing cancer were diagnosed with clinical finding, blood tests and imaging studies.

\section{Serum hepcidin measurement}

Serum hepcidin concentrations were determined using an enzyme-linked immunosorbent assay Kit for human hepcidin (Uscn Life Science Inc., Wuhan, China). The samples were assayed in duplicates.

\section{Ethics and informed consent for the patient study}

The ethics committee of Niigata University Medical and Dental Hospital approved the study, and all patients signed an informed consent form in relation to diagnosis by blood sample.

\section{Animal model}

Male Lewis rats were obtained from Charles River, Japan (Atsugi, Kanagawa, Japan), and were maintained in our experimental animal facilities until they reached 8 weeks of age. HF with LC was generated using monocrotaline (MCT) that causes pulmonary hypertension, as described in below. All animal experiments followed the guidelines for the care and use of laboratory animals published by the US National Institutes of Health and were done with the approval of Institutional Animal Care and Use Committee at the Niigata University. Rats $(n=37)$ were anesthetized with inhalation of isoflurane. Rats received a single subcutaneous injection of MCT (60 mg/ kg; Sigma Chemical, St. Louis, MO) and were studied 28 days (4 weeks, $n=11$ ), 35 days ( 5 weeks, $n=10$ ) and 42 days ( 6 weeks, $n=7$ ) after injection. Control rats received a subcutaneous injection of phosphate-buffered saline and studied ( 4 weeks, $n=3$; 5 weeks, $n=3$; 6 weeks, $n=3$ ). All animals were euthanized by an overdose of isoflurane inhalation in a killing chamber. Death of the animals was confirmed by the absence of breathing after removal of the carcass from the chamber and exsanguinated through the heart. Livers and hearts were collected for gene expression analyses and histological analyses.

\section{Pulse oximetry measurements}

Rats (Control, $n=6$; MCT, $n=14$ ) were weakly anesthetized with inhalation of isoflurane and arterial blood oxygen saturation was measured immediately in the footpad of the rats with a pulse oximeter (model 9847V; Nonin Medical, Plymouth, MN) and clip on sensor (2000SL; Nonin Medical) every week.

\section{Blood count}

Rat peripheral blood samples were obtained from the right atrium. Blood $\mathrm{Hb}$ concentrations were determined using the automated XE-2100 analyzer (Sysmex, Kobe, Japan).

\section{Serum iron measurement}

Rat serum iron and unsaturated iron-binding capacity were determined using an automated system H7700 (Hitachi High-Tech, Tokyo, Japan). The total iron-binding capacity was calculated as the sum of serum iron and unsaturated iron-binding capacity, and the percentage of transferrin saturation was calculated as (serum iron $\times 100$ )/ total iron-binding capacity. 


\section{Histology and Berlin blue staining}

Tissue samples were fixed at room temperature in $10 \%$ formalin. Samples from liver and heart were sequentially dehydrated through an alcohol series and embedded in paraffin. Four- $\mu \mathrm{m}$ thick sections were cut, deparaffinized in xylene, and dehydrated in descending series dilutions of ethanol. Each section from liver and heart was also stained with hematoxylin and eosin (HE) stain and Berlin blue stain for iron (Berlin blue staining set; WAKO Pure Chemical, Osaka, Japan).

\section{$R N A$ extraction and real time $R T-P C R$}

Total RNA was isolated from the liver tissues using Trizol (Invitrogen, Carlsbad, CA, USA). cDNA was synthesized from $5 \mu \mathrm{g}$ of total RNA with random primers and murine Moloney leukemia virus reverse transcriptase. To create the plasmids used for the standard, rat hepcidin was amplified using the primer pairs as reported previously (Isoda et al. 2010). PCR amplified cDNAs were inserted directly into the pGEM recombinant plasmids and were isolated following transformation into Escherichia coli JM109-competent cells using a MagExtractor plasmid kit (Toyobo, Osaka, Japan). The absolute copy number of each mRNA was also measured by quantitative real-time RT-PCR with a LightCycler instrument (Roche Diagnostics, Tokyo, Japan) using the same primers and SYBR Premix Ex Taq (Takara, Otsu, Japan). After an initial denaturation step of 10 minutes at $95^{\circ} \mathrm{C}$, a 3-step cycling procedure (denaturation at $95^{\circ} \mathrm{C}$ for $10 \mathrm{~s}$, annealing at $62^{\circ} \mathrm{C}$ for $10 \mathrm{~s}$ and extension at $72^{\circ} \mathrm{C}$ for $13 \mathrm{~s}$ ) was used for 45 cycles. The absolute copy numbers of particular transcripts were calculated by LightCycler software using a standard curve approach. Gene expressions of 5 samples in a rat liver preparation were averaged.

\section{Statistical analysis}

Statistical assessment was performed by an unpaired Student's t test or one-way analysis of variance (ANOVA) and Bonferroni's multiple comparison test. The differences were considered significant at $p<0.05$. The data obtained from organ weight, arterial blood saturation, blood examination, quantitative RT-PCR, and enzyme-linked immunosorbent assay were expressed as mean \pm standard error of the mean $(\mathrm{SEM})$ or mean \pm standard deviation. Correlations were evalu- ated by linear regression analysis. Data were analyzed using Pearson' s correlation coefficient and Fisher's Z-transformation test.

\section{Results}

Prevalence of anemia and relationship between anemia and $L C$ in all HF patients

Among consecutive HF patients admitted to the Cardiovascular Disease Department of Niigata University Medical and Dental Hospital, 41\% had anemia (Table 1). Plasma BNP levels were significantly higher in HF patients with anemia, but IVC diameter, an indicator of LC, was similar in HF patients, irrespective of anemia (Table 1). There was almost no correlation between log-plasma BNP levels and blood $\mathrm{Hb}$ concentrations in male (Fig. 1A) and female (Fig. 1B) HF patients. There was no significant correlation between IVC diameter and $\mathrm{Hb}$ concentrations in HF patients (Fig. 1D, E). Transferrin saturation (male, $n=$ 40; female, $n=15$ ) did not correlate to log-plasma BNP levels and IVC diameter in HF patients with anemia (Fig. 1C, F).

\section{Serum hepcidin level in HF patients with anemia}

We obtained 45 serum samples from patients with anemia and HF, who gave their consent and measured markers of HF by echocardiography, and compared markers of HF, anemia and renal function between LC and non-LC patients (Table 2). Plasma BNP levels were higher in patients with LC $($ IVC $\geq 2 \mathrm{~cm}$ ) than those without LC (IVC $<2 \mathrm{~cm}$ ), whereas serum hepcidin levels were similar in both groups of patients. Difference of serum creatinine levels between $\mathrm{LC}$ and non-LC patients is not significant.

We then explored the effects of LC or TRPG on the correlation between hepcidin levels and $\mathrm{Hb}$ concentrations (Fig. 2A, B). TRPG reflects pulmonary arterial pressure. There was a weak negative correlation between log-serum hepcidin levels and $\mathrm{Hb}$ concentrations in $19 \mathrm{LC}$ patients, whereas there was a weak positive correlation in 26 non-LC

Table 1. Characterization of heart failure patients (Clinical study 1).

\begin{tabular}{lccc}
\hline & $\begin{array}{c}\text { anemia } \\
n=131(41 \%)\end{array}$ & $\begin{array}{c}\text { non-anemia } \\
n=189(59 \%)\end{array}$ & $p$ value \\
\hline Age yrs & $72.2 \pm 11.0$ & $64.1 \pm 13.2$ & $<0.0001$ \\
Sex & & & \\
$\quad$ female & $28(28 \%)$ & $71(72 \%)$ & \\
$\quad$ male & $103(47 \%)$ & $118(53 \%)$ & 0.013 \\
BNP $(\mathrm{pg} / \mathrm{mL})$ & $743 \pm 1,091$ & $483 \pm 774$ & 0.145 \\
IVC diameter* $(\mathrm{cm})$ & $1.64 \pm 0.55$ & $1.54 \pm 0.52$ & \\
\hline
\end{tabular}

Values are mean \pm S.D. or $n(\%)$.

Consecutive patients with HF (BNP $>50 \mathrm{pg} / \mathrm{mL})$ were admitted to the Cardiovascular Disease Department of Niigata University Medical and Dental Hospital between August 2013 and July 2014. Anemia was defined as a $\mathrm{Hb}$ concentration below normal levels (male, $\mathrm{Hb}<13.2 \mathrm{~g} / \mathrm{dL}$; female, $\mathrm{Hb}<10.8 \mathrm{~g} / \mathrm{dL})$.

*IVC diameter of 255 patients (male 173, female 82; anemia 99, non-anemia 156) were measured. 

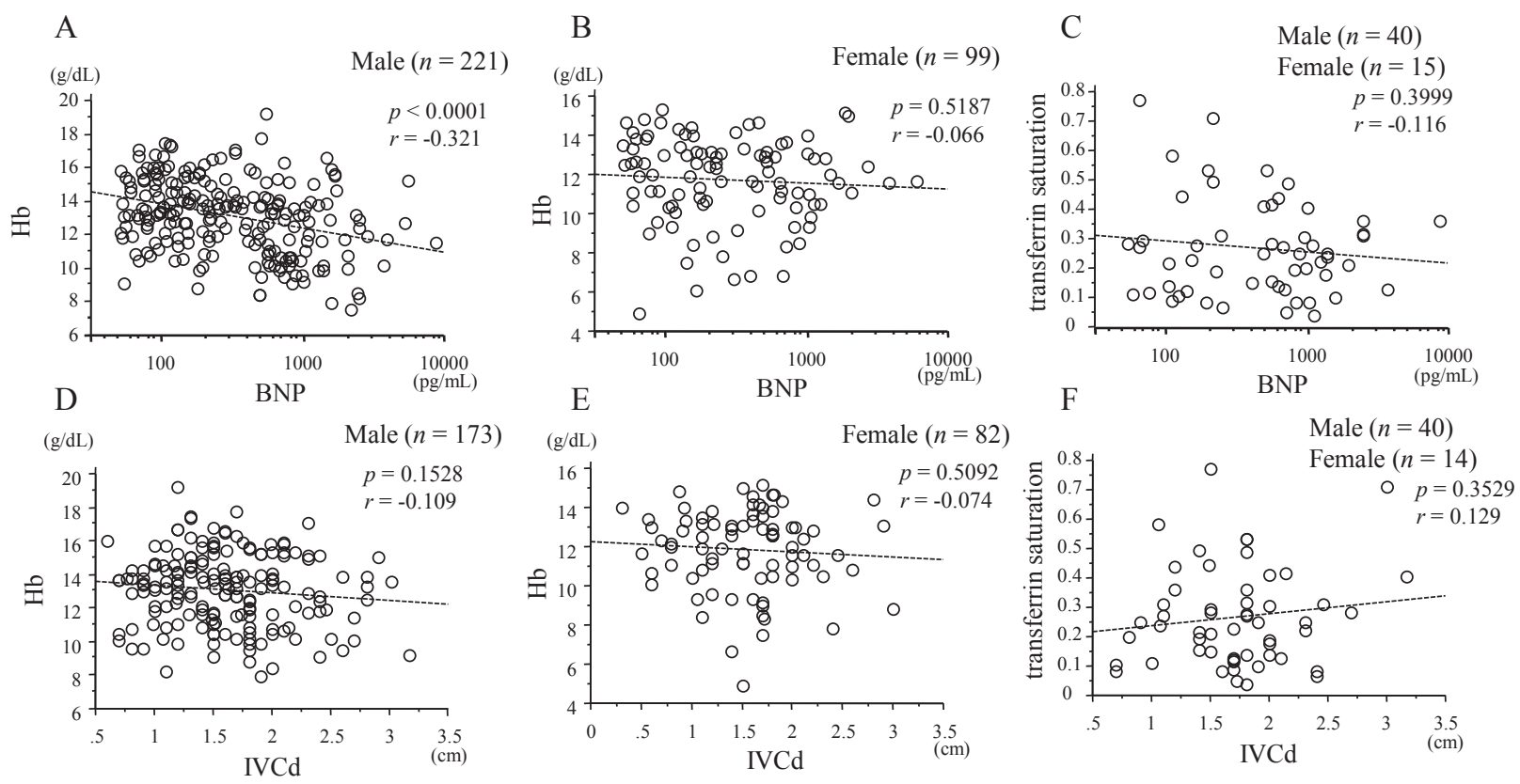

Fig. 1. Correlations between markers of HF and markers of anemia.

Characterization of HF patients is provided in Table 1. (A) Correlation between log-plasma BNP levels and Hb concentrations in male patients. (B) Correlation between log-plasma BNP levels and Hb concentrations in female patients. (C) Correlation between log-plasma BNP levels and transferring saturation. (D) Correlation between IVC diameter and $\mathrm{Hb}$ concentrations in male patients. (E) Correlation between IVC diameter and $\mathrm{Hb}$ concentrations in female patients. (F) Correlation between IVC diameter and transferring saturation.

patients (Fig. 2A upper panels). On the other hand, there was no correlation between log-serum hepcidin levels and $\mathrm{Hb}$ concentrations in patients with high TRPG or low TRPG (Fig. 2A lower panels). Subsequently, out of the 45 patients with anemia and HF, we excluded 15 patients with chronic diseases, such as active inflammation and non-curative cancer (LC, $n=1$ and non-LC, $n=14$ as shown in Table 2). The remaining 30 patients without chronic diseases were then analyzed (Fig. 2B). We again detected a weak negative correlation between log-serum hepcidin levels and $\mathrm{Hb}$ concentrations in $18 \mathrm{LC}$ patients and a weak positive correlation in 12 non-LC patients. On the other hand, there was no correlation between log-serum hepcidin levels and $\mathrm{Hb}$ concentrations, irrespective of TRPG. Thus, the correlations were similar to those detected in all patients $(n=45)$ (Fig. 2A). Moreover, log-serum hepcidin levels were higher in those patients with LC than those without LC (All, $p=0.0316 ; \mathrm{Hb} \leq 11, p=0.0100 ; \mathrm{Hb} \leq 10, p=$ 0.0168 ) (Fig. $2 \mathrm{C}$ left side graph). In contrast, no difference was detected in the log-serum hepcidin levels between the patients with high TRPG and those with low TRPG (Fig. $2 \mathrm{C}$ right side graph).

\section{Organ weight and histologic findings}

The heart weight/body weight ratio of MCT-treated rats gradually increased and the ratios of MCT-treated rats were significantly higher than that of control rats at all time points ( 4 weeks, $n=3 ; 5$ weeks, $n=3$; 6 weeks, $n=3$ ) (Fig. $3 \mathrm{~A})$. Marked right ventricular dilatation and hypertrophy of MCT-treated rats caused by pulmonary hypertension (PH) were observed from 4 wks to 6 wks (Fig. 4 left side panels). Microscopic examinations of livers of MCTtreated rats from 4 weeks to 6 weeks showed red blood cell accumulation in the sinusoidal spaces around the central vein (Fig. 4 middle panels) and several hemosiderin-loaded macrophages in Berlin blue stained sections (Fig. 4 right side panels), namely LC. The liver weight/body weight ratio of MCT-treated rats was increased with LC at 4 weeks. However, the ratio gradually decreased from 5 weeks to 6 weeks (Fig. 3B).

\section{Arterial blood oxygen saturation}

Arterial blood oxygen saturation levels of control rats were maintained at over $96 \%$. But those of MCT-treated rats gradually decreased to $82 \%$ at 6 weeks (Fig. 5).

\section{Anemia and serum iron in MCT-treated rats}

$\mathrm{Hb}$ and serum iron concentrations and transferrin saturation of MCT-treated rats decreased at 4-5 weeks (Fig. 6A-C). However, these parameters were recovered at 6 weeks.

\section{Expression of hepcidin}

The relative expression levels of hepcidin mRNA in livers of MCT-treated rats at 4 weeks were significantly higher than those in control rats, and those in livers of MCT-treated rats at 5 weeks were similar to the levels of control rats despite anemia and FID (Fig. 6D and squares 
Table 2. Characterization of heart failure patients with anemia (Clinical study 2).

\begin{tabular}{|c|c|c|c|}
\hline & $\begin{array}{c}\text { LC } \\
n=19(42 \%)\end{array}$ & $\begin{array}{c}\text { non-LC } \\
n=26(58 \%)\end{array}$ & $p$ value \\
\hline Age yrs & $63.7 \pm 12.5$ & $68.2 \pm 17.4$ & 0.343 \\
\hline \multicolumn{4}{|l|}{ Sex } \\
\hline female & $6(32 \%)$ & $7(27 \%)$ & \\
\hline male & $13(68 \%)$ & $19(73 \%)$ & \\
\hline \multicolumn{4}{|l|}{ Basic cardiac disease } \\
\hline Valvular heart disease & $8(42 \%)$ & $4(15 \%)$ & \\
\hline Congenital heart disease & $2(11 \%)$ & & \\
\hline Dilated cardiomyopathy & $5(26 \%)$ & $5(19 \%)$ & \\
\hline Hypertrophic cardiomyopathy & $1(5 \%)$ & $1(4 \%)$ & \\
\hline Cardiac amyloidosis & $1(5 \%)$ & & \\
\hline Ischemic heart disease & & $4(15 \%)$ & \\
\hline Hypertensive heart disease & & $4(15 \%)$ & \\
\hline Atrial fibrillation & & $1(4 \%)$ & \\
\hline Idiopathic ventricular tachycardia & & $1(4 \%)$ & \\
\hline Pulmonary hypertension & $2(11 \%)$ & $3(12 \%)$ & \\
\hline Cardiac tumor & & $1(4 \%)$ & \\
\hline Cardiac tamponade & & $1(4 \%)$ & \\
\hline Pericarditis & & $1(4 \%)$ & \\
\hline $\mathrm{Hb}(\mathrm{g} / \mathrm{dL})$ & $9.8 \pm 1.3$ & $10.5 \pm 1.3$ & 0.091 \\
\hline Cre (mg/dL) & $2.0 \pm 1.7$ & $2.4 \pm 3.2$ & 0.597 \\
\hline $\mathrm{BNP}(\mathrm{pg} / \mathrm{mL})$ & $1,138 \pm 1,560$ & $478 \pm 486$ & 0.048 \\
\hline ferritin* (ng/mL) & $196 \pm 246$ & $297 \pm 481$ & 0.409 \\
\hline hepcidin (ng/mL) & $169 \pm 196$ & $166 \pm 264$ & 0.9731 \\
\hline IVC diameter $(\mathrm{cm})$ & $2.43 \pm 0.28$ & $1.37 \pm 0.36$ & $<0.0001$ \\
\hline Chronic diseases such as active inflammation and non-curative cancer & $1(5 \%)$ & $14(54 \%)$ & \\
\hline
\end{tabular}

Values are mean \pm S.D. or $n(\%)$.

Consenting patients with HF (BNP $>50 \mathrm{pg} / \mathrm{mL})$ and anemia were admitted to the Cardiovascular Disease Department of

Niigata University Medical and Dental Hospital between 2008 and 2013.

Liver congestion (LC) was diagnosed by IVC diameter $(\geq 2.0 \mathrm{~cm})$ or clinical findings.

*Ferritin was measured for 42 patients (male 30, female 12; LC 19, non-LC 23).

and lozenges in Fig. 7). On the other hand, the levels at 6 weeks further decreased despite the recovery of anemia and FID (Fig. 6D and triangles in Fig. 7).

\section{Discussion}

The most common form of anemia is iron deficiency anemia, which is characterized by a reduction of hepcidin (such as iron deficiency anemia, and bleeding anemia). In contrast, the anemia of chronic disease is characterized by induction of hepcidin, which causes a degradation of ferroportin channels (De Domenico et al. 2007). Consequently, iron cannot be absorbed from the intestine, and is trapped within iron storage sites such as macrophages and hepatocytes.

\section{Anemia and hepcidin in HF patients}

Previous studies reported that $17-57 \%$ of patients admitted with HF had anemia (Horwich et al. 2002; Ezekowitz et al. 2003; Hamaguchi et al. 2009; Caira et al. 2013). In the present clinical study, the observed rate of anemia in consecutive HF patients admitted to the Cardiovascular Disease Department of Niigata University Medical and Dental Hospital was 41\%, which agreed with the previous reports. As previously reported (Horwich et al. 2002; Tsuji et al. 2004), plasma BNP levels of HF patients with anemia were significantly higher than those without anemia. Anemia independently predicts substantially increased risks of death and hospitalization in HF (Mozaffarian et al. 2003; Go et al. 2006). These data show that clinicians should attend to anemia in medical care of HF patients as is widely proposed.

Firstly, we evaluated the relation between anemia and LC in all HF patients with anemia. But in this study, the IVC diameter, an indicator of LC, of HF patients with anemia were not significantly larger than those without anemia, and $\mathrm{Hb}$ concentrations were not correlated to the severity of LC. These results suggest that LC-associated anemia may occur in a minority of cases with anemia.

Secondly, we evaluated the influence of LC on the serum hepcidin level that may reflect hepcidin production. 

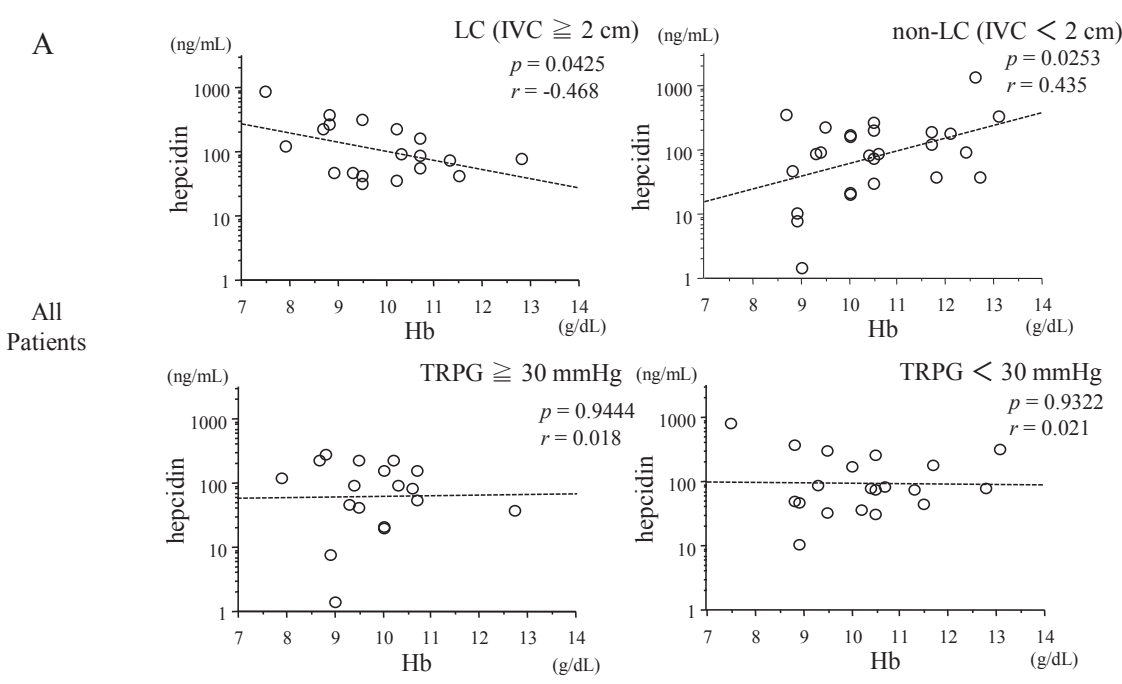

B
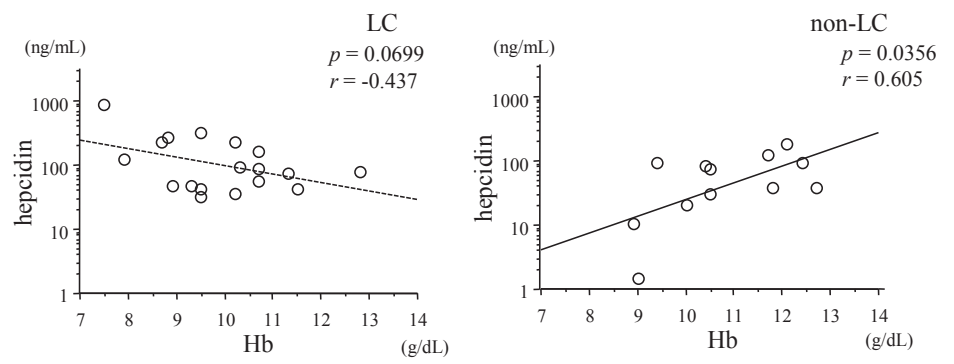

Without

Chronic

Disease
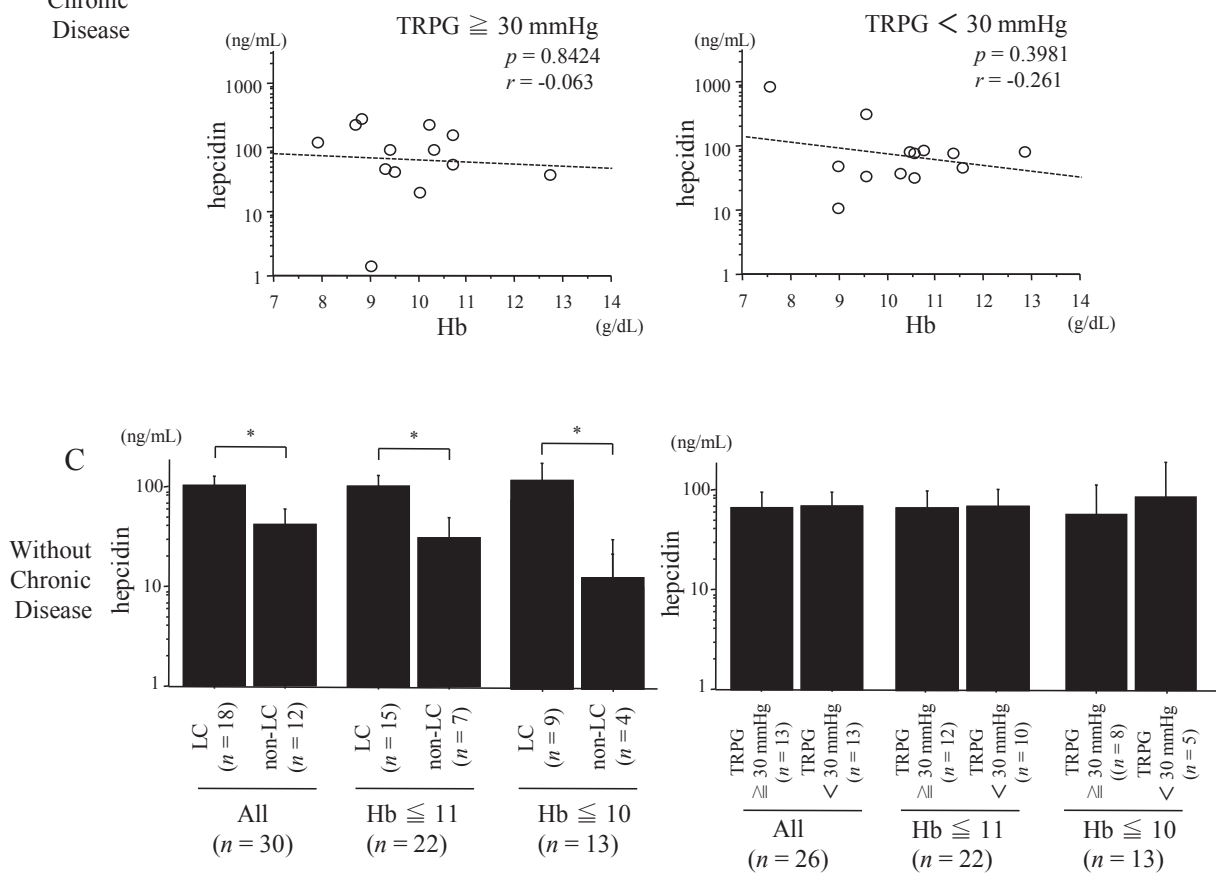

Fig. 2. Serum hepcidin concentrations in patients with HF.

Characterization of HF patients with anemia is provided in Table 2. (A) Correlation between log-serum hepcidin levels and $\mathrm{Hb}$ concentrations in all $\mathrm{HF}$ patients with anemia (LC, $n=19$; non-LC, $n=26$ ) (TRPG $\geq 30 \mathrm{mmHg}, n=18$; TRPG $<30 \mathrm{mmHg}, n=20$ ). Upper panels, difference between LC (IVC $\geq 2 \mathrm{~cm}$ ) (left) and non-LC (IVC $<2 \mathrm{~cm})$ (right) patients; and lower panels, difference between TRPG $\geq 30 \mathrm{mmHg}$ (left) and TRPG $<30 \mathrm{mmHg}$ (right) patients. (B) Correlation between log-serum hepcidin levels and $\mathrm{Hb}$ concentrations in patients without chronic disease (LC, $n=18$; nonLC, $n=12$ ) (TRPG $\geq 30 \mathrm{mmHg}, n=13$; TRPG $<30 \mathrm{mmHg}, n=13$ ). Upper panels, difference between LC (IVC $\geq 2$ $\mathrm{cm}$ ) (left) and non-LC (IVC $<2 \mathrm{~cm}$ ) (right) patients; and lower panels, difference between TRPG $\geq 30 \mathrm{mmHg}$ (left) and TRPG $<30 \mathrm{mmHg}$ (right) patients. (C) Serum hepcidin concentrations in HF patients without chronic disease defined by $\mathrm{Hb}$ levels. Left panel, difference between LC and non-LC patients; and right panel, difference between TRPG $\geq 30$ $\mathrm{mmHg}$ and TRPG $<30 \mathrm{mmHg}$ patients. Error bar represents SEM. Statistical analysis was performed by an unpaired Student's t test. $* p<0.05$. 

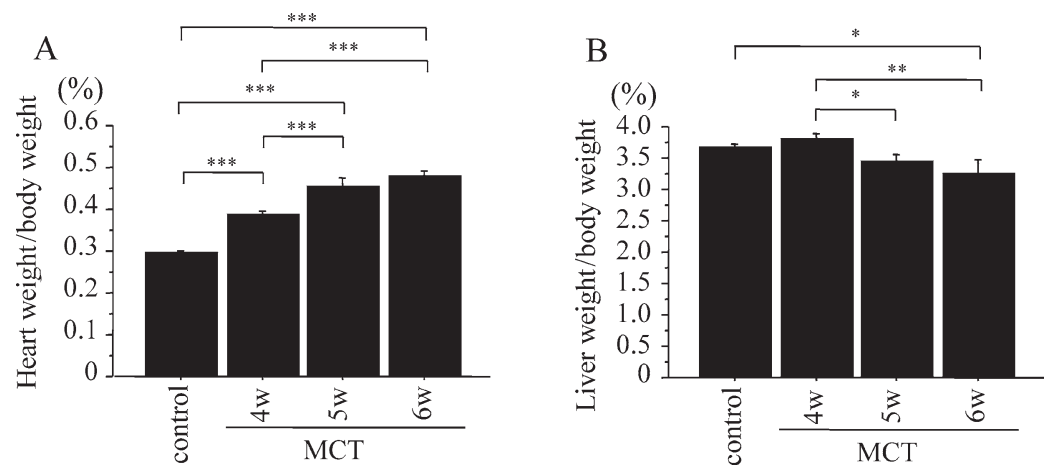

Fig. 3. Organ weight of MCT-treated rats.

(A) Heart weight/body weight ratio. (B) Liver weight/body weight ratio. Error bar represents SEM. Statistical analysis was performed by one-way ANOVA and Bonferroni's multiple comparison test. ${ }^{*} p<0.05,{ }^{*} p<0.01$, $* * * p<0.001$. Control rats $(n=9)$ were injected with phosphate buffered saline ( 4 weeks, $n=3 ; 5$ weeks, $n=3$; 6 weeks, $n=3$ ).
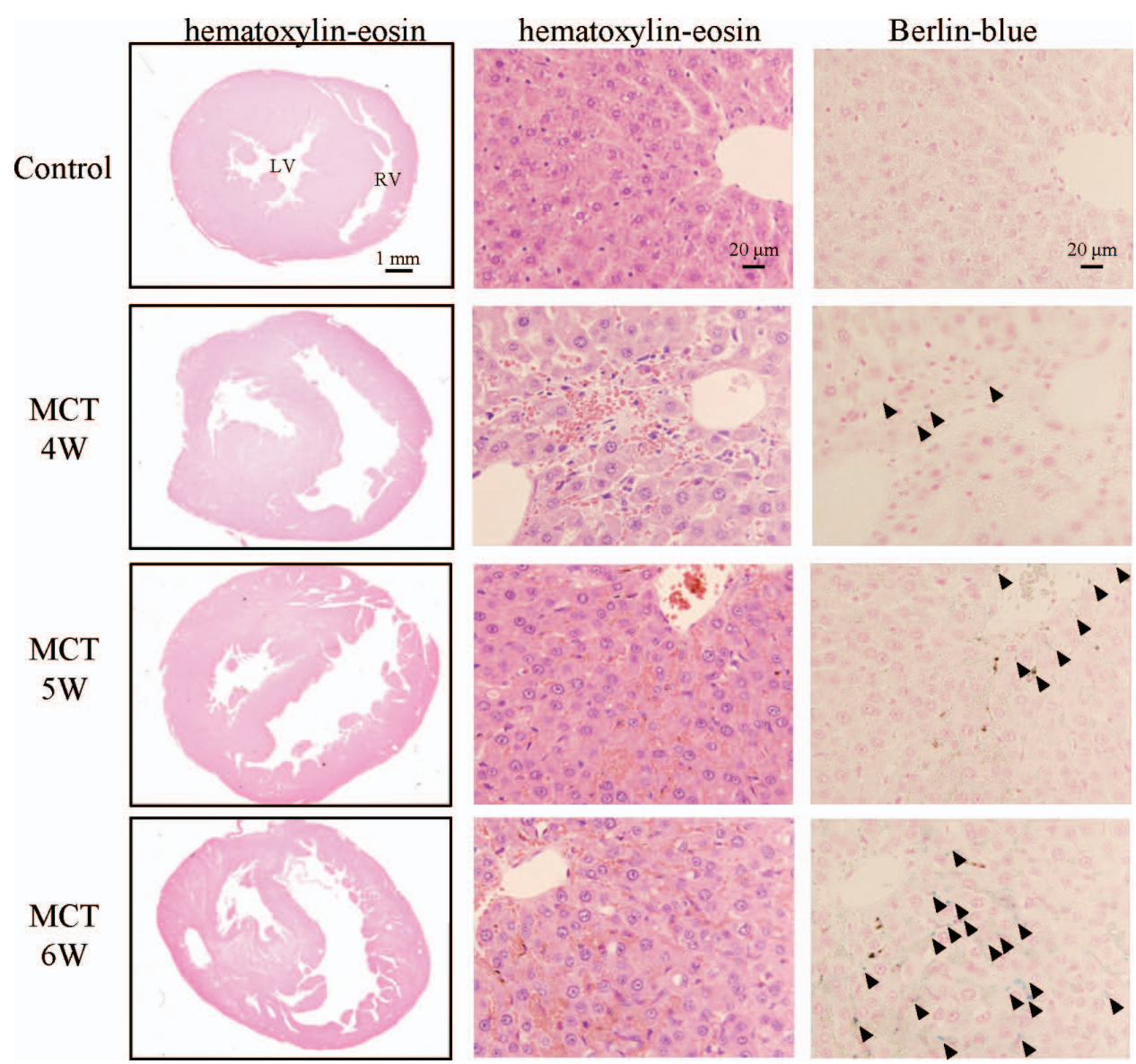

Fig. 4. Microscopic finding.

Tissue sections were stained with HE and Berlin blue for iron. Left panels, sections stained with HE from hearts of a control rat at 4 weeks and MCT-treated rats; Middle panels, sections stained with HE from livers of a control rat at 4 weeks and MCT-treated rats; Right panels, sections stained with Berlin blue from livers of a control rat at 4 weeks and MCT-treated rats. Arrow heads indicate hemosiderin-loaded cells, which were faintly stained by Berlin-blue. LV, left ventricle; RV, right ventricle.

$\mathrm{Hb}$ concentrations correlated positively with serum hepcidin levels in patients without LC. This phenomenon has also been observed in children with iron deficiency (Choi et al. 2012), the most common form of iron deficiency anemia, which is characterized by a reduction of hepcidin. In contrast, $\mathrm{Hb}$ concentrations in LC patients correlated negatively with hepcidin levels, which is a type of anemia characterized by induction of hepcidin. Moreover, because hepcidin is induced by chronic disease including infections, malignancies, and rheumatologic disorders (Weinstein et al. 


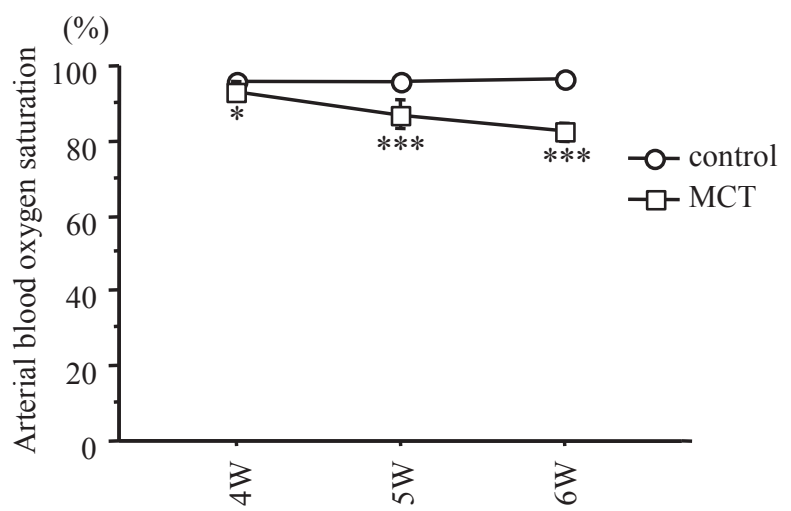

Fig. 5. Arterial blood oxygen saturation.

Open circles represent control rats and open squares represent MCT-treated rats. Error bar represents standard deviation. Statistical analysis was performed by an unpaired Student's t test. ${ }^{*} p<0.05$ vs. control rats, ${ }^{* * *} p$ $<0.001$ vs. control rats.

2002; Weiss and Goodnough 2005; Maes et al. 2010), we analyzed serum hepcidin levels in HF and anemia patients without chronic disease. In this series of HF patients without chronic disease, serum hepcidin levels were higher in LC patients than those in patients without LC; namely, hepcidin levels of LC patients did not decrease despite low $\mathrm{Hb}$ levels. These results suggest inappropriately increased hepcidin production in LC patients.

Anemia, FID and hepcidin expression in MCT-treated PAH rats

Histological findings of liver in MCT-treated rats at 4-6 weeks showed congestion. In MCT-treated rats at 4-5 weeks, $\mathrm{Hb}$ and serum iron concentration and transferrin saturation were decreased and the expression levels of hepcidin in livers were not decreased despite anemia and FID. These findings were similar to those of rats with LC by ligating the IVC as our previously reported (Suzuki et al. 2014) and suggest inappropriately increased expression of hepcidin, that is, a form of anemia characterized by an induction of hepcidin. On the other hand, MCT-treated rats at 6 weeks were quite different from rats with LC by ligating the IVC (Suzuki et al. 2014). Liver weight/body weight ratio in LC rats in which the IVC was ligated was persistently high and with severe continuing LC throughout the course of ligation, while, in contrast, liver weight/body weight ratio in MCT-treated rats at 6 weeks decreased and liver atrophy was observed. We surmised that the liver atrophy observed in this model was due to the unusual and severe hypoxemia and low cardiac output (Denis et al. 2004; Farahani et al. 2008). Previous studies have reported that hypoxia reduced hepcidin expression in hepatocytes (Nicolas et al. 2002). Moreover, the low cardiac output causes hypoxia in several organs and tissues (Fahey and Lister 1989); therefore, the low cardiac output may regulate hepcidin expression in a similar way as hypoxemia. In fact, hepcidin expression of MCT-treated rats at 6 weeks
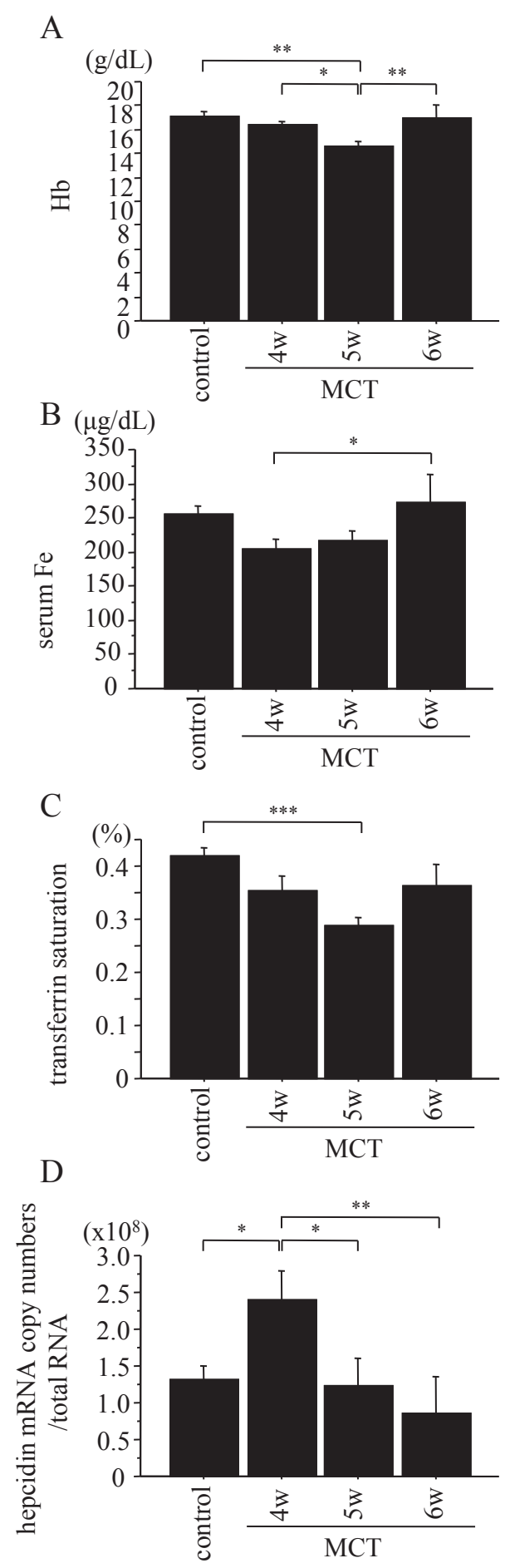

Fig. 6. Anemia and iron markers in control rats and MCTtreated rats.

(A) Blood Hb concentrations. (B) Serum iron concentrations. (C) Transferring saturation. (D) Hepcidin mRNA level in the liver. Hepcidin expression levels of 5 samples in a rat liver preparation were averaged. Error bar represents SEM. Statistical analysis was performed by one-way ANOVA and Bonferroni's multiple comparison test. $* p<0.05, * * p<0.01, * * * p<0.001$. Control rats $(n=9)$ were injected with phosphate buffered saline (4 weeks, $n=3$; 5 weeks, $n=3$; 6 weeks, $n=3$ ). 


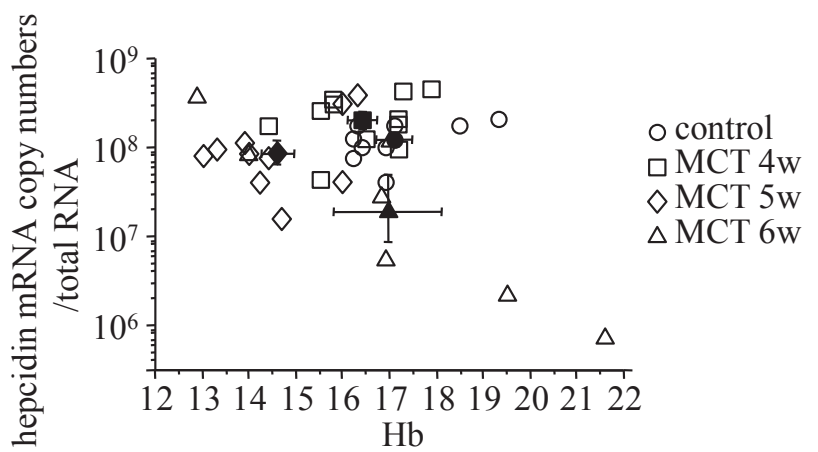

Fig. 7. Relationship between $\mathrm{Hb}$ concentrations and hepcidin expression.

Relationship between $\mathrm{Hb}$ concentrations and log-hepcidin expression levels in control rats (circles) and MCTtreated rats at 4 weeks (squares), 5 weeks (lozenges) and 6 weeks (triangles). Solid symbols indicate mean levels in each group. Error bar represents SEM.

decreased; therefore, the decrease is likely due to hypoxemia and low cardiac output.

The most common findings of the liver in clinical right HF patients are not atrophy but congestion. In fact, LC is frequently associated with $\mathrm{PAH}$, mitral stenosis, tricuspid regurgitation, cor pulmonale, constrictive pericarditis, cardiac amyloidosis, or some types of congenital heart disease (Alvarez and Mukherjee 2011), whereas right HF with liver atrophy is rare (Greenberg and Kahn 2012). Our reported case of tricuspid regurgitation (Suzuki et al. 2012) also had persistent LC, refractory anemia, and a very high serum hepcidin level, but did not have severe hypoxemia and the low cardiac output. In such a case with persistent LC, inappropriate increased hepcidin expression seems to be caused by LC and results in anemia and FID.

On the other hand, findings of MCT-treated rats at 6 weeks suggest that hypoxemia and the low cardiac output, also observed in HF patients, are important in iron metabolism. Clinical PAH patients with hypoxemia are often treated by oxygen inhalation (Palmisano et al. 1990), and congenital heart disease with right-to-left shunt flow often causes severe hypoxemia (Webb et al. 2012) and severe left HF with lung congestion (Gheorghiade et al. 2012) leading to hypoxemia and the low cardiac output. Hypoxemia results in secondary polycythemia (Webb et al. 2012) and increased iron absorption (Simpson 1996; Mastrogiannaki et al. 2013). In this study, MCT-treated rats showed severe hypoxemia at 6 weeks and the anemia and FID later recovered unlike LC rats in which the IVC was ligated. Anyway, in HF patients, various factors influence hepcidin expression, such as LC, hypoxemia, and low cardiac output; these factors should be also considered for understanding iron metabolism. However, there is a limitation of this animal study; namely, the MCT-treated rat model is not a typical model of clinical right HF patients with LC.

In conclusion, LC complicating HF with anemia induces hepcidin production, which may worsen the degree of anemia and FID. However, this study was conducted in a limited number of HF patients with anemia. Additional studies are needed to confirm the results in a large number of HF patients with anemia. Recently, the effects of intravenous iron therapy for anemia and iron deficiency of HF patients have been studied (Anker et al. 2009a, b). Other investigators have been developing anti-hepcidin drugs (Cooke et al. 2013; Schwoebel et al. 2013). Future studies are needed to classify anemia and FID of HF patients based on etiology and to establish proper therapies for anemia and FID of HF patients.

\section{Acknowledgments}

This study was supported in part by a Grant for scientific research from the Ministry of Education, Culture, Sports, Science and Technology of Japan (No. 25461047).

\section{Conflict of Interest}

The authors declare no conflict of interest.

\section{References}

Alvarez, A.M. \& Mukherjee, D. (2011) Liver abnormalities in cardiac diseases and heart failure. Int. J. Angiol., 20, 135-142.

Androne, A.S., Katz, S.D., Lund, L., LaManca, J., Hudaihed, A., Hryniewicz, K. \& Mancini, D.M. (2003) Hemodilution is common in patients with advanced heart failure. Circulation, 107, 226-229.

Anker, S.D., Colet, J.C., Filippatos, G., Willenheimer, R., Dickstein, K., Drexler, H., Luscher, T.F., Mori, C., von Eisenhart Rothe, B., Pocock, S., Poole-Wilson, P.A. \& Ponikowski, P.; FAIRHF committees and investigators (2009a) Rationale and design of Ferinject assessment in patients with IRon deficiency and chronic Heart Failure (FAIR-HF) study: a randomized, placebo-controlled study of intravenous iron supplementation in patients with and without anaemia. Eur. J. Heart Fail., 11, 1084-1091.

Anker, S.D., Comin Colet, J., Filippatos, G., Willenheimer, R., Dickstein, K., Drexler, H., Luscher, T.F., Bart, B., Banasiak, W., Niegowska, J., Kirwan, B.A., Mori, C., von Eisenhart Rothe, B., Pocock, S.J., Poole-Wilson, P.A., et al. (2009b) Ferric carboxymaltose in patients with heart failure and iron deficiency. N. Engl. J. Med., 361, 2436-2448.

Arndt, S., Maegdefrau, U., Dorn, C., Schardt, K., Hellerbrand, C. \& Bosserhoff, A.K. (2010) Iron-induced expression of bone morphogenic protein 6 in intestinal cells is the main regulator of hepatic hepcidin expression in vivo. Gastroenterology, 138, 372-382.

Caira, C., Ansalone, G., Mancone, M., Canali, E., Pagliaro, M., Fratarcangeli, L., Aznaran, C.A., Gatto, M.C., Di Pietro, R. \& Fedele, F. (2013) Heart failure and iron deficiency anemia in Italy: results from CARMES-1 registry. Future Cardiol., 9, 437-444.

Choi, H.S., Song, S.H., Lee, J.H., Kim, H.J. \& Yang, H.R. (2012) Serum hepcidin levels and iron parameters in children with iron deficiency. Korean J. Hematol., 47, 286-292.

Cooke, K.S., Hinkle, B., Salimi-Moosavi, H., Foltz, I., King, C., Rathanaswami, P., Winters, A., Steavenson, S., Begley, C.G., Molineux, G. \& Sasu, B.J. (2013) A fully human antihepcidin antibody modulates iron metabolism in both mice and nonhuman primates. Blood, 122, 3054-3061.

De Domenico, I., Ward, D.M. \& Kaplan, J. (2007) Hepcidin regulation: ironing out the details. J. Clin. Invest., 117, 1755-1758.

Denis, C., De Kerguennec, C., Bernuau, J., Beauvais, F. \& Cohen Solal, A. (2004) Acute hypoxic hepatitis ('liver shock'): still a 
frequently overlooked cardiological diagnosis. Eur. J. Heart Fail., 6, 561-565.

Eschbach, J.W. (2002) Anemia management in chronic kidney disease: role of factors affecting epoetin responsiveness. $J$. Am. Soc. Nephrol., 13, 1412-1414.

Ezekowitz, J.A., McAlister, F.A. \& Armstrong, P.W. (2003) Anemia is common in heart failure and is associated with poor outcomes: insights from a cohort of 12065 patients with newonset heart failure. Circulation, 107, 223-225.

Fahey, J.T. \& Lister, G. (1989) Response to low cardiac output: developmental differences in metabolism during oxygen deficit and recovery in lambs. Pediatr. Res., 26, 180-187.

Farahani, R., Kanaan, A., Gavrialov, O., Brunnert, S., Douglas, R.M., Morcillo, P. \& Haddad, G.G. (2008) Differential effects of chronic intermittent and chronic constant hypoxia on postnatal growth and development. Pediatr. Pulmonol., 43, 20-28.

Fleming, R.E. \& Ponka, P. (2012) Iron overload in human disease. N. Engl. J. Med., 366, 348-359.

Ganz, T. (2003) Hepcidin, a key regulator of iron metabolism and mediator of anemia of inflammation. Blood, 102, 783-788.

Gheorghiade, M., Filippatos, G.S. \& Felker, G.M. (2012) Diagnosis and management of acute heart failure syndromes. In Braunwald's Heart Disease, 9th ed., edited by Bonow, R.O., Mann, D.L., Zipes, D.P., Libby, P. \& Braunwald, E. Elsevier, Phliadelphia, PA, pp. 517-542.

Go, A.S., Yang, J., Ackerson, L.M., Lepper, K., Robbins, S., Massie, B.M. \& Shlipak, M.G. (2006) Hemoglobin level, chronic kidney disease, and the risks of death and hospitalization in adults with chronic heart failure: the Anemia in Chronic Heart Failure: Outcomes and Resource Utilization (ANCHOR) Study. Circulation, 113, 2713-2723.

Greenberg, B. \& Kahn, A.M. (2012) Clinical assessment of heart failure. In Braunwald's Heart Disease, 9th ed., edited by Bonow, R.O., Mann, D.L., Zipes, D.P., Libby, P. \& Braunwald, E. Elsevier, Phliadelphia, PA, pp. 505-516.

Hamaguchi, S., Tsuchihashi-Makaya, M., Kinugawa, S., Yokota, T., Takeshita, A., Yokoshiki, H. \& Tsutsui, H.; JCARE-CARD Investigators (2009) Anemia is an independent predictor of long-term adverse outcomes in patients hospitalized with heart failure in Japan. A report from the Japanese Cardiac Registry of Heart Failure in Cardiology (JCARE-CARD). Circ. J., 73, 1901-1908.

Holleran, G., Hall, B., Hussey, M. \& McNamara, D. (2013) Small bowel angiodysplasia and novel disease associations: a cohort study. Scand. J. Gastroenterol., 48, 433-438.

Horwich, T.B., Fonarow, G.C., Hamilton, M.A., MacLellan, W.R. \& Borenstein, J. (2002) Anemia is associated with worse symptoms, greater impairment in functional capacity and a significant increase in mortality in patients with advanced heart failure. J. Am. Coll. Cardiol., 39, 1780-1786.

Isoda, M., Hanawa, H., Watanabe, R., Yoshida, T., Toba, K., Yoshida, K., Kojima, M., Otaki, K., Hao, K., Ding, L., Tanaka, K., Takayama, T., Kato, K., Okura, Y., Kodama, M., et al. (2010) Expression of the peptide hormone hepcidin increases in cardiomyocytes under myocarditis and myocardial infarction. J. Nutr. Biochem., 21, 749-756.

Jankowska, E.A., Malyszko, J., Ardehali, H., Koc-Zorawska, E., Banasiak, W., von Haehling, S., Macdougall, I.C., Weiss, G., McMurray, J.J., Anker, S.D., Gheorghiade, M. \& Ponikowski, P. (2013) Iron status in patients with chronic heart failure. Eur. Heart J., 34, 827-834.

Kliger, C., Eiros, R., Isasti, G., Einhorn, B., Jelnin, V., Cohen, H., Kronzon, I., Perk, G., Fontana, G.P. \& Ruiz, C.E. (2013) Review of surgical prosthetic paravalvular leaks: diagnosis and catheter-based closure. Eur. Heart J., 34, 638-649.

Macdougall, I.C., Canaud, B., de Francisco, A.L., Filippatos, G., Ponikowski, P., Silverberg, D., van Veldhuisen, D.J. \& Anker, S.D. (2012) Beyond the cardiorenal anaemia syndrome: recognizing the role of iron deficiency. Eur. J. Heart Fail., 14,
$882-886$

Maes, K., Nemeth, E., Roodman, G.D., Huston, A., Esteve, F., Freytes, C., Callander, N., Katodritou, E., Tussing-Humphreys, L., Rivera, S., Vanderkerken, K., Lichtenstein, A. \& Ganz, T. (2010) In anemia of multiple myeloma, hepcidin is induced by increased bone morphogenetic protein 2. Blood, 116, 3635-3644.

Mastrogiannaki, M., Matak, P. \& Peyssonnaux, C. (2013) The gut in iron homeostasis: role of HIF-2 under normal and pathological conditions. Blood, 122, 885-892.

Matsumoto, M., Tsujino, T., Lee-Kawabata, M., Naito, Y., Akahori, H., Sakoda, T., Ohyanagi, M., Tomosugi, N. \& Masuyama, T. (2010) Iron regulatory hormone hepcidin decreases in chronic heart failure patients with anemia. Circ. J., 74, 301-306.

Mozaffarian, D., Nye, R. \& Levy, W.C. (2003) Anemia predicts mortality in severe heart failure: the prospective randomized amlodipine survival evaluation (PRAISE). J. Am. Coll. Cardiol., 41, 1933-1939.

Nemeth, E., Valore, E.V., Territo, M., Schiller, G., Lichtenstein, A. \& Ganz, T. (2003) Hepcidin, a putative mediator of anemia of inflammation, is a type II acute-phase protein. Blood, 101, 2461-2463.

Nicolas, G., Chauvet, C., Viatte, L., Danan, J.L., Bigard, X., Devaux, I., Beaumont, C., Kahn, A. \& Vaulont, S. (2002) The gene encoding the iron regulatory peptide hepcidin is regulated by anemia, hypoxia, and inflammation. J. Clin. Invest., 110, 1037-1044.

Opasich, C., Cazzola, M., Scelsi, L., De Feo, S., Bosimini, E., Lagioia, R., Febo, O., Ferrari, R., Fucili, A., Moratti, R., Tramarin, R. \& Tavazzi, L. (2005) Blunted erythropoietin production and defective iron supply for erythropoiesis as major causes of anaemia in patients with chronic heart failure. Eur. Heart J., 26, 2232-2237.

Palmisano, J.M., Martin, J.M., Krauzowicz, B.A., Truman, K.H. \& Meliones, J.N. (1990) Effects of supplemental oxygen administration in an infant with pulmonary artery hypertension. Heart Lung, 19, 627-630.

Rhodes, C.J., Howard, L.S., Busbridge, M., Ashby, D., Kondili, E., Gibbs, J.S., Wharton, J. \& Wilkins, M.R. (2011) Iron deficiency and raised hepcidin in idiopathic pulmonary arterial hypertension: clinical prevalence, outcomes, and mechanistic insights. J. Am. Coll. Cardiol., 58, 300-309.

Schwoebel, F., van Eijk, L.T., Zboralski, D., Sell, S., Buchner, K., Maasch, C., Purschke, W.G., Humphrey, M., Zollner, S., Eulberg, D., Morich, F., Pickkers, P. \& Klussmann, S. (2013) The effects of the anti-hepcidin Spiegelmer NOX-H94 on inflammation-induced anemia in cynomolgus monkeys. Blood, 121, 2311-2315.

Simpson, R.J. (1996) Dietary iron levels and hypoxia independently affect iron absorption in mice. J. Nutr., 126, 18581864.

Suzuki, T., Hanawa, H., Ding, L., Ito, M., Kashimura, T., Obata, H., $\mathrm{Hu}$, Y.H., Jiao, S., Namura, O. \& Kodama, M. (2012) Improvement of anemia with decreasing hepcidin levels following valve replacement for severe tricuspid regurgitation. Eur. J. Haematol., 88, 551-552.

Suzuki, T., Hanawa, H., Jiao, S., Ohno, Y., Hayashi, Y., Yoshida, K., Kashimura, T., Obata, H. \& Minamino, T. (2014) Inappropriate expression of hepcidin by liver congestion contributes to anemia and relative iron deficiency. J. Card. Fail., 20, 268-277.

Tsuji, H., Nishino, N., Kimura, Y., Yamada, K., Nukui, M., Yamamoto, S., Iwasaka, T. \& Takahashi, H. (2004) Haemoglobin level influences plasma brain natriuretic peptide concentration. Acta Cardiol., 59, 527-531.

van der Meer, P., Lok, D.J., Januzzi, J.L., de la Porte, P.W., Lipsic, E., van Wijngaarden, J., Voors, A.A., van Gilst, W.H. \& van Veldhuisen, D.J. (2008) Adequacy of endogenous erythropoietin levels and mortality in anaemic heart failure patients. Eur. 
Heart J., 29, 1510-1515.

Webb, G.D., Smallhorn, J.F., Therrien, J. \& Redington, A.N. (2012) Congenital heart disease. In Braunwald's Heart Disease, 9th ed., edited by Bonow, R.O., Mann, D.L., Zipes, D.P., Libby, P. \& Braunwald, E. Elsevier, Phliadelphia, PA, pp. 1411-1467.

Weinstein, D.A., Roy, C.N., Fleming, M.D., Loda, M.F., Wolfsdorf, J.I. \& Andrews, N.C. (2002) Inappropriate expression of hepcidin is associated with iron refractory anemia: implications for the anemia of chronic disease. Blood, 100, 37763781 .
Weiss, G. \& Goodnough, L.T. (2005) Anemia of chronic disease. N. Engl. J. Med., 352, 1011-1023.

Westenbrink, B.D., Voors, A.A., de Boer, R.A., Schuringa, J.J., Klinkenberg, T., van der Harst, P., Vellenga, E., van Veldhuisen, D.J. \& van Gilst, W.H. (2010) Bone marrow dysfunction in chronic heart failure patients. Eur. J. Heart Fail., 12, 676-684.

Witte, K.K., Desilva, R., Chattopadhyay, S., Ghosh, J., Cleland, J.G. \& Clark, A.L. (2004) Are hematinic deficiencies the cause of anemia in chronic heart failure? Am. Heart J., 147, 924-930. 